\title{
Diverse Routing in Networks with Star SRLGs
}

\author{
Early stage research
}

\author{
Jean-Claude Bermond, David Coudert, Gianlorenzo D’Angelo, Fatima Zahra Moataz ${ }^{\dagger}$ \\ Mascotte Project - INRIA, I3S, CNRS, Univ. Nice Sophia, 2004 route des Lucioles 06560 Sophia-Antipolis, France \\ e-mail: \{jean-claude.bermond, david.coudert, gianlorenzo.d_angelo, fatima-zahra.moataz\}@inria.fr
}

\begin{abstract}
The notion of Shared Risk Link Group, SRLG has been introduced to capture multiple correlated failures in a network. A SRLG is a set of links that fail simultaneously if a given event (risk) occurs. In such multiple failures scenario, the problem of Diverse Routing consists in finding two SRLGdisjoint paths between a pair of nodes. We consider such problem for localized failures, when all the links of a SRLG verify the star property i.e. when they are incident to the same node. We prove that in this case the problem is in general NP-complete and determine some polynomial cases.
\end{abstract}

\section{Categories and Subject Descriptors}

G.2.2 [Discrete Mathematics]: Graph theory-Graph algorithms, Network problems; F.2.0 [Analysis of Algorithms and Problem Complexity]: General

\section{Keywords}

Diverse routing, SRLG

Motivations. To ensure reliable communications, many protection schemes have been proposed. One of the most used, called dedicated path protection, consists in computing for each demand both a working and a protection path. A general requirement is that these paths have to be diversely routed, so that at least one path can survive a single failure in the network. This method works well in a single link failure scenario, as it consists in finding two edge-disjoint paths between a pair of nodes. This is a well-known problem in graph theory for which there exist efficient polynomial algorithms. However, the problem of finding two diversely routed paths between a pair of nodes becomes much more difficult in case of multiple correlated link failures that can be captured by the notion of SRLG (Shared Risk Link

\footnotetext{
${ }^{*}$ Full version of this work is available in [1].

${ }^{\dagger}$ The results presented in this abstract are part of the Master thesis of the fourth author.
}

Permission to make digital or hard copies of all or part of this work for personal or classroom use is granted without fee provided that copies are not made or distributed for profit or commercial advantage and that copies bear this notice and the full citation on the first page. To copy otherwise, to republish, to post on servers or to redistribute to lists, requires prior specific permission and/or a fee.

CoNEXT Student'12, December 10, 2012, Nice, France.

Copyright 2012 ACM 978-1-4503-1779-5/12/12 ...\$15.00.
Group). In fact, a SRLG is a set of network links that fail simultaneously if a given event (i.e. risk) occurs. The scope of this concept is very broad. For example it can correspond to a set of fiber links of an optical backbone network that are physically buried at the same location and so that could be cut simultaneously (i.e. backhoe or JCB fade). It can also represent links that are located in the same seismic area, or radio links in access and backhaul networks subject to localized environmental conditions affecting signal transmission, or traffic jam propagation in road networks. In practice the failures are localized and very common SRLGs are star SRLGs or SRLGs verifying the star property [7] (coincident SRLGs in [5]). Under this property, a link can be affected by more than one risk but all links of any SRLG share an endpoint. Such failure scenarios can correspond to risks arising in router nodes like card failures or to the cut of a conduit containing links issued from a node (see Figure 1).
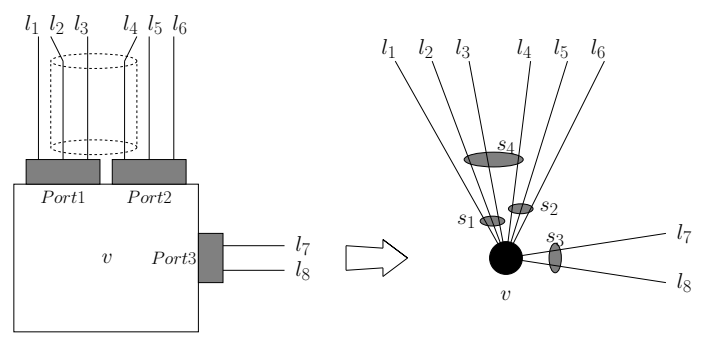

Figure 1: An example of localized risks. Link $l_{4}$, for instance, shares risk $s_{2}$, corresponding to Port 2 failure, with $l_{5}$ and $l_{6}$ and shares risk $s_{4}$, corresponding to the conduit cut, with links $l_{2}$ and $l_{3}$.

Related work. In the context of SRLG, basic network connectivity problems have been proven much more difficult to address than their usual counterparts. For instance, the problem of finding a "SRLG-shortest" st-path that is a path from node $s$ to node $t$ having the minimum number of risks has been proven $N P$-hard and hard to approximate in general (see [2]). However, the problem has been proven to be polynomial in two generic practical cases corresponding to localized failures: when all risks verify the star property [3] and when risks are of span 1; i.e. when a link is affected by at most one risk and links sharing a given risk form a connected component [2].

The diverse routing problem has been proven $N P$-complete in general (see [1] for references) and many heuristics have been proposed. The problem has been proven to be polynomial in some specific cases of localized failures: when SRLGs 
have span 1 [2], and in a specific case of SRLG's having the star property [4] (this result also follows from [2]).

The more general case of diverse routing with the star property where there are no restrictions on the number of risks per link has been studied in [7]. The authors claim that the diverse routing problem can be solved in polynomial time. Unfortunately their algorithm is not correct; indeed we exhibit counterexamples showing that in some cases their algorithm concludes to the non existence of two SRLGdisjoint paths although two such paths exist. We have not only disproved the validity of their algorithm, but have also been able to prove that the problem is in fact NP-complete (again, contradicting the supposed polynomiality of the algorithm, unless $P=N P$ ) and we have also determined some cases where the diverse routing problem is polynomial.

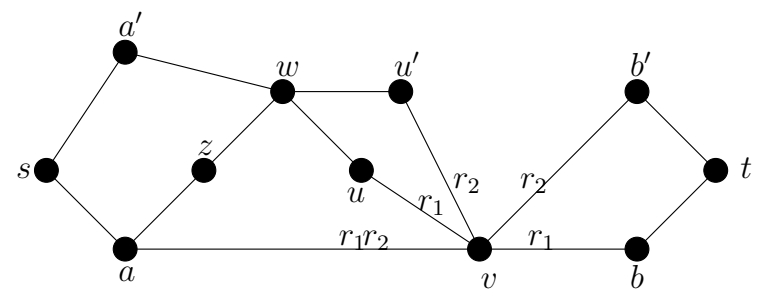

Figure 2: Example

Our precise results. Figure 2 shows a counter example to the algorithm in [7] that can give to the reader a flavor of the difficulty of the problem. In this figure, we have 2 specific risks $r_{1}$ and $r_{2} \neq r_{1}$ forming a star in $v$. Other risks are not represented, we can either suppose there are no other or that they are different from the risks explicitly mentioned and appear on only one link. As vertex $v$ is a cutvertex any path should contain $v$. In this case, and to ensure the SRLG-disjointedness, one path should use the subpath $u, v, b$ and the other one should use the subpath $u^{\prime}, v, b^{\prime}$. We have two SRLG-disjoint paths $P_{1}=\{s, a, z, w, u, v, b, t\}$ and $P_{2}=\left\{s, a^{\prime}, w, u^{\prime}, v, b^{\prime}, t\right\}$. The algorithm of [7] uses the shortest path $P_{a}=\{s, a, v, b, t\}$ and then performs a backwards phase which never finds $w$ again. Then the algorithm stops missing the fact that there exist two SRLG-disjoint paths (for details see the full version [1]).

To state precisely our results we use the colored-graph model $[2,7,8]$, also called labeled-graph model. In the colored-graph model, the network is modeled by a graph $G=(V, E)$ and the set of SRLGs by a set of colors $\mathcal{R}$. Each SRLG is modeled by a distinct color and an edge modeling a network link subject to different SRLGs will be assigned as many colors as SRLGs. In such a model, the star property corresponds to the fact that all the edges with the same color are incident to a common vertex and so form a star. In what follows we denote by $\Delta$ the maximum degree, and by $\Delta_{c}$ the maximum colored degree (the number of colors incident to a node).

As main results we show that finding 2 color-disjoint paths in a colored graph (i.e. two SRLG-disjoint paths) with the star property is $N P$-complete and more generally finding $k \geq 2$ color-disjoint paths (for a fixed $k$ ) is also $N P$ complete. This last result holds even if $\Delta$ is fixed with $\Delta \geq 6+k$ or if the number of colors per arc is at most 2 or the maximum number of arcs having the same color is at most 2; these values correspond to real networks parameters.
On the positive side, we show that the diverse routing problem can be solved in polynomial time in particular subcases which are relevant in practice. Namely, we solve the problem when the input graph is a DAG, when the number of SRLGs is bounded by a constant or when the maximum degree $\Delta \leq 4$. The algorithm for DAGs requires $O\left((|V| \Delta)^{2 k}\right)$ time where $k$ is the number of color-disjoint paths. The algorithm for fixed $|\mathcal{R}|$ and the one for $\Delta \leq 4$ require $O(|V|+|E|)$ time.

Finally we also consider the problem of finding the maximum number of color-disjoint paths in a colored graph. This problem has been shown to be $N P$-hard in [6]. We prove that it is also $N P$-hard in colored graphs with the star property even if the number of colors per arc is at most 3 or the maximum number of arcs having the same color is at most 2 . Moreover, unless $P=N P$, such problem cannot be approximated within $O(|V|)$ even if either the number of colors per arc is fixed or if the maximum number of arcs having the same color is fixed. Such inapproximability results hold also when the graph is a Directed Acyclic Graph (DAG).

These results, along with the polynomial cases, give an almost complete characterization of the problem of finding color-disjoint paths in networks with the star property.

\section{References}

[1] J.-C. Bermond, D. Coudert, G. D'Angelo, and F. Z. Moataz. Diverse Routing with the star property. Research Report RR-8071, INRIA, September 2012.

[2] D. Coudert, P. Datta, S. Perennes, H. Rivano, and M-E. Voge. Shared risk resource group: Complexity and approximability issues. Parallel Processing Letters, 17(2):169-184, June 2007.

[3] D. Coudert, S. Perennes, H. Rivano, and M-E. Voge. Shared risk resource groups and colored graph: Polynomial cases and transformation issues. Technical Report inria-00175143, HAL, September 2007.

[4] P. Datta and A.K. Somani. Graph transformation approaches for diverse routing in shared risk resource group (SRRG) failures. Computer Networks, 52(12):2381-2394, August 2008.

[5] J. Doucette and W. Grover. Shared-risk logical span groups in span-restorable optical networks: Analysis and capacity planning model. Photonic Network Communications, 9(1):35-53, 2005.

[6] Li G., Kalmanek C., and Doverspike R. Fiber span failure protection in mesh optical networks. Optical Networks Magazine, pages 21-31, May 2002.

[7] X. Luo and B. Wang. Diverse routing in WDM optical networks with shared risk link group (SRLG) failures. In Proc. 5th DRCN, pages 445-452. IEEE, October 2005.

[8] S. Yuan, S. Varma, and J.P. Jue. Minimum-color path problems for reliability in mesh networks. In Proc. IEEE INFOCOM, volume 4, pages 2658-2669, Houston, TX, USA, March 2005. 